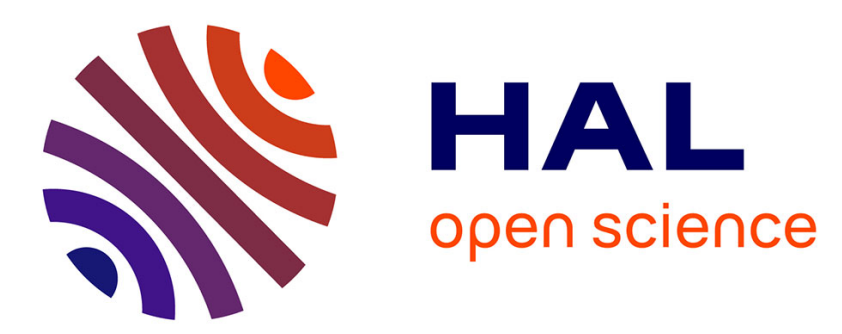

\title{
Towards Optimal Bounds on the Recoverable Strains in Polycrystalline Shape Memory Alloys
}

\author{
Michaël Peigney
}

\section{To cite this version:}

Michaël Peigney. Towards Optimal Bounds on the Recoverable Strains in Polycrystalline Shape Memory Alloys. M. Frémond and F.Maceri. Mechanics, Models and Methods in Civil Engineering, Springer, pp.175-184, 2012, 978-3-642-24638-8. 10.1007/978-3-642-24638-8_10 . hal-01133551

\section{HAL Id: hal-01133551 \\ https://hal-enpc.archives-ouvertes.fr/hal-01133551}

Submitted on 19 Mar 2015

HAL is a multi-disciplinary open access archive for the deposit and dissemination of scientific research documents, whether they are published or not. The documents may come from teaching and research institutions in France or abroad, or from public or private research centers.
L'archive ouverte pluridisciplinaire HAL, est destinée au dépôt et à la diffusion de documents scientifiques de niveau recherche, publiés ou non, émanant des établissements d'enseignement et de recherche français ou étrangers, des laboratoires publics ou privés. 


\title{
Towards optimal bounds on the recoverable strains in polycrystalline shape memory alloys
}

\author{
Michaël Peigney*
}

\begin{abstract}
The set of recoverable (or stress-free) strains plays a central role in the peculiar properties of shape memory alloys. New upper bounds are presented for estimating the recoverable strains of polycrystalline shape memory alloys, in the finite strains setting. Those bounds take the texture of the polycrystal (i.e. the shapes, distribution and orientations of the grains) into account. A reference two-orientation problem is studied for investigating the optimality of the bounds proposed.
\end{abstract}

Key words: Phase transformation, Polycrystals, Finite strain, Nonlinear bounds

\section{Introduction}

Some metallic alloys (such as TiNi or CuAlNi) are known to display a shape memory behaviour : after cooling down a sample and deforming it mechanically, the sample recovers its initial shape after heating. This shape memory effect is the result of a solid/solid phase transformation between different crystallographic structures, known as austenite (stable at high temperature) and martensite (stable at low temperature). In terms of crystallographic structure, the austenite has a higher symmetry than the martensite. Therefore, the martensite actually exists in the form of several variants, corresponding to different orientations of the martensitic lattice with respect to the austenitic lattice. Accordingly, to each martensitic variant is attached a transformation strain, describing the deformation between the crystallographic structures of the austenite and the martensite. The number of martensitic variants as well as the corresponding transformation strains depend on the alloys considered (see e.g. Bhattacharya (2003) for some examples and additional details).

Email address: michael.peigney@polytechnique.org (Michaël Peigney). 
The shape memory effect is explained as follows: cooling down a stress-free sample transforms the homogeneous austenite to a martensitic microstructure, in which the martensitic variants arrange themselves so as to produce a stressfree state with no macroscopic deformation. This phenomenon is classically referred to as self-accommodation. Applying a mechanical loading entails a reorientation of the variants. When the loading is removed, there remains a macroscopic residual deformation, which is achieved by a cooperative effect of the transformation strains in each variant. Heating the sample transforms the martensite back in austenite, thus restoring the initial configuration.

The shape memory effect is obviously limited: if the strain imposed in the cooled state is too large, plasticity will occur and the material will no longer be able to recover its initial shape after heating. A macroscopic strain will be called recoverable if it can be restored by the shape memory effect. This article is concerned with the theoretical prediction of the (bounded) set of recoverable strains. Some experimental measurements of maximum uniaxial recoverable strains are displayed in Table 1 (see Bhattacharya and Kohn (1997) and references therein).

\begin{tabular}{|c|lll|}
\hline & $\mathrm{Ni}-37 \mathrm{Al}$ & $\mathrm{Cu}-14 \mathrm{Al}-4 \mathrm{Ni}$ & $\mathrm{Ti}-50.6 \mathrm{Ni}$ \\
\hline single crystal & $0-13 \%$ & $2-9 \%$ & $3-10 \%$ \\
\hline polycrystal & $0.7 \%$ & $2.5 \%$ (uncontrolled texture) & $4-8 \%$ \\
& & $6.5 \%$ (special texture) & \\
\hline
\end{tabular}

Table 1 - Measurements of maximum uniaxial recoverable strains

Two remarks are in order. The first one is that recoverable strains can be relatively large (up to 10\%). This means that a study in finite strains (rather than infinitesimal strains) as to be preferred. The second remark is that large differences are observed between single crystals and polycrystals. Understanding those differences is one of the goals of this article: assuming that the set of recoverable strains of the single crystal is known, we aim at predicting the set of recoverable strains of a polycrystal, taking the influence of the texture (i.e. the shape, distribution and orientation of the grains) into account. Such investigation has notably been carried out by Bhattacharya and Kohn (1997) as well as Shu and Bhattacharya (1998) in the context of infinitesimal strains (i.e. in the geometrically linear theory). The originality here is that we deal with the finite strain setting (i.e. geometrically nonlinear theory), consistently with the experimental observations. Let us insist on the fact that the results in Table 1 are uniaxial measurements, carried out along prescribed orientations. The material is generally not isotropic, and the maximum uniaxial recoverable strain depends on the loading direction. Therefore, recoverable strains cannot be completely characterized by a scalar giving a uniaxial maximum value: the 
set of recoverable strains has to be considered as a (bounded) domain in the space of deformation gradients $\mathbb{R}^{3 \times 3}$.

\section{Problem considered}

We consider a polycrystal of shape memory alloy, occupying a domain $\Omega$ in the reference configuration. The texture of the polycrystal is described by characteristic functions $\chi_{r}(r=1, \cdots, n)$, such that the domain $\Omega^{r}=\{\boldsymbol{x} \in$ $\left.\Omega \mid \chi_{r}(\boldsymbol{x})=1\right\}$ is occupied by grains with the same orientation relative to a reference single crystal. That orientation is described by a rotation $\boldsymbol{R}_{r}$. The characteristic functions $\chi_{r}$ verify $\chi_{r}(\boldsymbol{x}) \in\{0,1\}$ and $\sum_{r=1}^{n} \chi_{r}(\boldsymbol{x})=1$ for all $\boldsymbol{x} \in \Omega$.

Let $\mathcal{S}_{0} \subset \mathbb{R}^{3 \times 3}$ be the set of recoverable strains of the reference single crystal: when submitted to a large transformation $\boldsymbol{u}(\boldsymbol{x})$, a reference single crystal displays the shape memory effect as long as the deformation gradient $\boldsymbol{F}=$ $\nabla \boldsymbol{u}(\boldsymbol{x})$ remains in $\mathcal{S}_{0}$. That set $\mathcal{S}_{0}$ is determined by the number of martensitic variants as well as their respective transformation strains, and is assumed to be known. The set $\mathcal{S}_{0}$ is closed and bounded, but not necessarily convex. Elements $\boldsymbol{F}$ of $\mathcal{S}_{0}$ notably verify the material impenetrability condition $\operatorname{det} \boldsymbol{F}>0$. Because of the principle of frame indifference, $\mathcal{S}_{0}$ can be written as

$$
\mathcal{S}_{0}=\mathrm{SO}(3) \mathcal{U}_{0}
$$

where $\mathrm{SO}(3)$ is the group or rotations and $\mathcal{U}_{0}$ is included in the set of symmetric positive tensors $\mathbb{R}_{+}^{3 \times 3}$. The decomposition (2.1) means that $\mathcal{S}_{0}$ only depends on the deformation gradient $\boldsymbol{F}$ through the right-stretch tensor $\boldsymbol{U}$ of its polar decomposition $\boldsymbol{F}=\boldsymbol{R U}$.

Let $\tilde{\mathcal{S}}$ be the set of macroscopic (or effective) recoverable strains for the polycrystal. Choosing length units such that the domain $\Omega$ has unit volume, $\tilde{\mathcal{S}}$ is defined mathematically by

$$
\tilde{\mathcal{S}}=\{\overline{\boldsymbol{F}} \mid \exists \boldsymbol{u}(\boldsymbol{x}) \text { such that } \boldsymbol{u}(\boldsymbol{x})=\overline{\boldsymbol{F}} \cdot \boldsymbol{x} \text { on } \partial \Omega ; \nabla \boldsymbol{u}(\boldsymbol{x}) \in \mathcal{S}(\boldsymbol{x}) \text { in } \Omega\}
$$

where

$$
\mathcal{S}(\boldsymbol{x})=\sum_{r=1}^{n} \chi_{r}(\boldsymbol{x}) \boldsymbol{R}_{r}^{T} \mathcal{S}_{0} \boldsymbol{R}_{r}
$$

Estimating $\tilde{\mathcal{S}}$ is essentially a problem of geometric compatibility, consisting in finding the effective deformation gradients that can be achieved by transformation $\boldsymbol{u}(\boldsymbol{x})$ which are subjected to a local constraint on $\nabla \boldsymbol{u}(\boldsymbol{x})$. The principle 
of frame indifference implies that $\tilde{\mathcal{S}}$ can be written as

$$
\tilde{\mathcal{S}}=\operatorname{SO}(3) \tilde{\mathcal{U}}
$$

where $\tilde{\mathcal{U}}$ is a subset of $\mathbb{R}_{+}^{3 \times 3}$.

\section{Upper bounds on $\tilde{\mathcal{S}}$}

Recently, a general methodology for deriving upper bounds on $\tilde{\mathcal{S}}$ has been proposed (Peigney, 2008), following an original homogenization approach firstly introduced by Milton and Serkov (2000) and latted refined by Talbot and Willis (2004), Peigney (2005). That methodology makes use of the so-called translation method, and is now briefly summarized. As detailled in Peigney (2008), the indicator function $I_{\mathcal{\mathcal { S }}}(\overline{\boldsymbol{F}})$ of $\tilde{\mathcal{S}}$ (equal to 0 if $\overline{\boldsymbol{F}}$ is in $\tilde{\mathcal{S}}$, and infinite otherwise) verifies the inequality

$$
I_{\tilde{\mathcal{S}}}(\overline{\boldsymbol{F}}) \geq \sup _{\boldsymbol{\tau}} \inf _{\boldsymbol{F} \in \mathcal{K}(\overline{\boldsymbol{F}})} \int_{\Omega}\left\{\boldsymbol{F}: \boldsymbol{\tau}+U(\boldsymbol{F} ; \boldsymbol{x})-\left(I_{\mathcal{S}}-U\right)^{*}(\boldsymbol{\tau} ; \boldsymbol{x})\right\} d \boldsymbol{x}
$$

where

$$
\mathcal{K}(\overline{\boldsymbol{F}})=\{\boldsymbol{F} \mid \operatorname{det} \boldsymbol{F}>0, \exists \boldsymbol{u}(\boldsymbol{x}) \text { such that } \boldsymbol{F}=\nabla \boldsymbol{u} \text { in } \Omega ; \boldsymbol{u}(\boldsymbol{x})=\overline{\boldsymbol{F}} . \boldsymbol{x} \text { on } \partial \Omega\}
$$

and

$$
\left(I_{\mathcal{S}}-U\right)^{*}(\boldsymbol{\tau} ; \boldsymbol{x})=\sup _{\boldsymbol{F} \in \mathcal{S}(\boldsymbol{x})} \boldsymbol{F}: \boldsymbol{\tau}+U(\boldsymbol{F} ; \boldsymbol{x})
$$

In (3.4), the function $U: \mathbb{R}^{3 \times 3} \times \Omega \mapsto \mathbb{R}$ is arbitrary and refered to as a comparison potential. The second-order tensor $\boldsymbol{\tau}$ in (3.4) is also arbitrary and refered to as a polarization. Any choice of comparison potential and polarization thus generates a lower bound on $I_{\tilde{\mathcal{S}}}$. Such a lower bound automatically translates into an upper bound on $\tilde{\mathcal{S}}$. Indeed, let $I^{-}(\overline{\boldsymbol{F}})$ be the right-hand side of (3.4). Since any $\overline{\boldsymbol{F}}$ in $\tilde{\mathcal{S}}$ verifies $I_{\tilde{\mathcal{S}}}(\overline{\boldsymbol{F}})=0$, we have

$$
\tilde{\mathcal{S}} \subset\left\{\overline{\boldsymbol{F}} \mid I^{-}(\overline{\boldsymbol{F}}) \leq 0\right\}
$$

The set $\left\{\overline{\boldsymbol{F}} \mid I^{-}(\overline{\boldsymbol{F}}) \leq 0\right\}$ is thus an upper bound (in the sense of inclusion of sets) on $\tilde{\mathcal{S}}$. For such bounds to be relevant, the comparison potential and the polarization need to be chosen in such a way that the right-hand side of (3.4) is finite. Since the set $\mathcal{S}(\boldsymbol{x})$ is closed and bounded, it is clear that $\left(I_{\mathcal{S}}-U\right)^{*}(\boldsymbol{\tau} ; \boldsymbol{x})$ is finite for all choice of $\boldsymbol{U}(\boldsymbol{F} ; \boldsymbol{x})$ continuous in $\boldsymbol{F}$. However, this does not necessarily prevent the infimum in (3.4) for being equal to $-\infty$. Moreover, even in the case where $I^{-}(\overline{\boldsymbol{F}})$ is finite, the infimum problem in 
(3.4) may prove to be difficult to solve if $U$ and $\boldsymbol{\tau}$ are not chosen carefully. A possibility explored in Peigney (2008) is to use a potential $U$ of the form

$$
U(\boldsymbol{F} ; \boldsymbol{x})=\boldsymbol{a}: \boldsymbol{F}^{*}+\alpha \operatorname{det} \boldsymbol{F}
$$

where $\boldsymbol{a}$ and $\alpha$ are arbitrary, and $\boldsymbol{F}^{*}$ is the adjugate of $\boldsymbol{F}$, defined by $\boldsymbol{F}^{*}=$ $\operatorname{det} \boldsymbol{F}\left(\boldsymbol{F}^{-1}\right)^{T}$. Such potentials $U$ are homogeneous, i.e. independent on $\boldsymbol{x}$. In the following, we will drop the dependence with respect to $\boldsymbol{x}$ in the notation, thus simply writing $U(\boldsymbol{F})$ instead of $U(\boldsymbol{F} ; \boldsymbol{x})$. Functions of the form (3.6) are known to be null-lagrangians (Dacorogna, 1989), which implies that they satisfy the following equality:

$$
U(\overline{\boldsymbol{F}}) \leq \inf _{\boldsymbol{F} \in \mathcal{K}(\overline{\boldsymbol{F}})} \int_{\Omega} U(\boldsymbol{F}) d \boldsymbol{x}
$$

Moreover, for potentials $U$ of the form (3.6), it can be calculated that

$$
\left(I_{\mathcal{S}}-U\right)^{*}(\boldsymbol{\tau} ; \boldsymbol{x})=\sum_{r=1}^{n} \chi_{r}(\boldsymbol{x}) f_{r}(\boldsymbol{a}, \boldsymbol{\tau}, \alpha)
$$

where

$$
f_{r}(\boldsymbol{a}, \boldsymbol{\tau}, \alpha)=\sup _{\boldsymbol{U} \in \boldsymbol{R}_{r}^{T} \mathcal{U}_{0} \boldsymbol{R}^{r}} \lambda_{1}+\lambda_{2}+\operatorname{sgn}(\operatorname{det} \boldsymbol{M}) \lambda_{3}+\alpha \operatorname{det} \boldsymbol{U}
$$

In (3.8), $\boldsymbol{M}$ is defined by $\boldsymbol{M}=\boldsymbol{\tau} \boldsymbol{U}+\boldsymbol{a} \boldsymbol{U}^{*}$ and $\left(\lambda_{1}, \lambda_{2}, \lambda_{3}\right)$ are the singular values of the tensor $\operatorname{sgn}(\operatorname{det} \boldsymbol{M}) \boldsymbol{M}$, ordered in such a way that $\lambda_{1} \geq \lambda_{2} \geq \lambda_{3}$. Observe also that any $\boldsymbol{F} \in \mathcal{K}(\overline{\boldsymbol{F}})$ satisfies

$$
\overline{\boldsymbol{F}}=\int_{\Omega} \boldsymbol{F}(\boldsymbol{x}) d \boldsymbol{x}
$$

Substituting the relations (3.6)-(3.9) in (3.4), we obtain

$$
\tilde{\mathcal{S}} \subset\left\{\overline{\boldsymbol{F}} \mid 0 \geq \sup _{\boldsymbol{\tau}} \boldsymbol{\tau}: \overline{\boldsymbol{F}}+\boldsymbol{a}: \overline{\boldsymbol{F}}^{*}+\alpha \operatorname{det} \overline{\boldsymbol{F}}-\sum_{r=1}^{n} c_{r} f_{r}(\boldsymbol{\tau}, \boldsymbol{a}, \alpha)\right\}
$$

where $c_{r}=\int_{\Omega} \chi_{r}(\boldsymbol{x}) d \boldsymbol{x}$. The right-hand side of (3.10) is an explicit upper bound on $\tilde{\mathcal{S}}$. The inclusion (3.10) holds for any $\boldsymbol{a}$ and $\alpha$. Consequently, we have

$$
\tilde{\mathcal{S}} \subset \mathcal{S}^{+}(\boldsymbol{c})
$$

where

$$
\mathcal{S}^{+}(\boldsymbol{c})=\bigcap_{\boldsymbol{\tau}, \boldsymbol{a}, \alpha}\left\{\overline{\boldsymbol{F}} \mid 0 \geq \boldsymbol{\tau}: \overline{\boldsymbol{F}}+\boldsymbol{a}: \overline{\boldsymbol{F}}^{*}+\alpha \operatorname{det} \overline{\boldsymbol{F}}-\sum_{r=1}^{n} c_{r} f_{r}(\boldsymbol{\tau}, \boldsymbol{a}, \alpha)\right\}
$$

The upper bound $\mathcal{S}^{+}(\boldsymbol{c})$ only depends on the functions $\chi_{r}$ through the volume fractions $\boldsymbol{c}=\left(c_{1}, \cdots, c_{n}\right)$. In other words, that upper bound only involves onepoint statistics of the texture. It can easily be verified that $\mathcal{S}^{+}(\boldsymbol{c})$ respects the 
frame indifference, and therefore can be written as

$$
\mathcal{S}^{+}(\boldsymbol{c})=\mathrm{SO}(3) \mathcal{U}^{+}(\boldsymbol{c})
$$

where $\mathcal{U}^{+}(\boldsymbol{c})$ is a subset of $\mathbb{R}_{+}^{3 \times 3}$.

\section{A reference two-orientation problem}

In finite strains, very few analytical results are available concerning recoverable strains of martensitic crystals. For a single crystal with two martensitic variants, the set of recoverable strains has been determined by Ball and James (1992). With an appropriate choice of the reference basis $\left\{\boldsymbol{v}_{1}, \boldsymbol{v}_{2}, \boldsymbol{v}_{3}\right\}$, the set obtained by Ball and James (1992) consists of tensors $\boldsymbol{F}$ such that

$$
\boldsymbol{F}^{T} . \boldsymbol{F}=\left(\begin{array}{ccc}
c_{11} & c_{12} & 0 \\
c_{12} & c_{22} & 0 \\
0 & 0 & 1
\end{array}\right) \text { with } c_{11} c_{22}-c_{12}^{2}=1 ; 0 \leq c_{11} \leq 1+\delta^{2} ; c_{22} \leq 1
$$

where $\delta$ is a given parameter. The matrix representation in (4.13) is relative to the basis $\left(\boldsymbol{v}_{1}, \boldsymbol{v}_{2}, \boldsymbol{v}_{3}\right)$. In the following, the set defined by (4.13) is used as the set of recoverable deformation gradients $\mathcal{S}_{0}$ for the reference single crystal.

We consider a polycrystalline material with only two orientations $\boldsymbol{R}_{1}$ and $\boldsymbol{R}_{2}$. The rotation $\boldsymbol{R}_{1}$ is taken as the identity, and $\boldsymbol{R}_{2}$ is the rotation of angle $\pi / 4$ around $\boldsymbol{v}_{3}$. The sets $\mathcal{S}_{1}=\mathcal{S}_{0}$ and $\mathcal{S}_{2}=\boldsymbol{R}_{2}^{T} \mathcal{S}_{0} \boldsymbol{R}_{2}$ can be written as $\mathcal{S}_{1}=\mathrm{SO}(3) \mathcal{U}_{1}, \mathcal{S}_{2}=\mathrm{SO}(3) \mathcal{U}_{2}$ where $\mathcal{U}_{1}=\mathcal{U}_{0}$ and $\mathcal{U}_{2}=\boldsymbol{R}_{2}^{T} \mathcal{U}_{0} \boldsymbol{R}_{2}$. The definitions of $\mathcal{S}_{1}$ and $\mathcal{S}_{2}$ imply that any second-order tensor $\boldsymbol{U}$ in $\mathcal{U}_{1}$ or $\mathcal{U}_{2}$ is of the form

$$
\boldsymbol{U}=u_{11} \boldsymbol{v}_{1} \otimes \boldsymbol{v}_{1}+u_{22} \boldsymbol{v}_{2} \otimes \boldsymbol{v}_{2}+u_{12}\left(\boldsymbol{v}_{1} \otimes \boldsymbol{v}_{2}+\boldsymbol{v}_{2} \otimes \boldsymbol{v}_{1}\right)+\boldsymbol{v}_{3} \otimes \boldsymbol{v}_{3}
$$

with $u_{11} u_{22}-u_{12}^{2}=1$. More precisely, tensors $\boldsymbol{U}$ in $\mathcal{U}_{1}$ satisfy

$$
\frac{1+u_{12}^{2}}{\sqrt{1-u_{12}^{2}}} \leq u_{11} \leq \sqrt{1+\delta^{2}-u_{12}^{2}}
$$

whereas tensors in $\mathcal{U}_{2}$ satisfy

$$
u_{11}=\frac{1}{2}\left(u_{11}^{\prime}+2 u_{12}^{\prime}+\frac{1-u_{12}^{\prime 2}}{u_{11}^{\prime}}\right), u_{12}=\frac{1}{2}\left(\frac{1-u_{12}^{\prime 2}}{u_{11}^{\prime}}-u_{11}^{\prime}\right)
$$

for some $\left(u_{12}^{\prime}, u_{11}^{\prime}\right)$ in $\mathcal{U}_{1}$. 


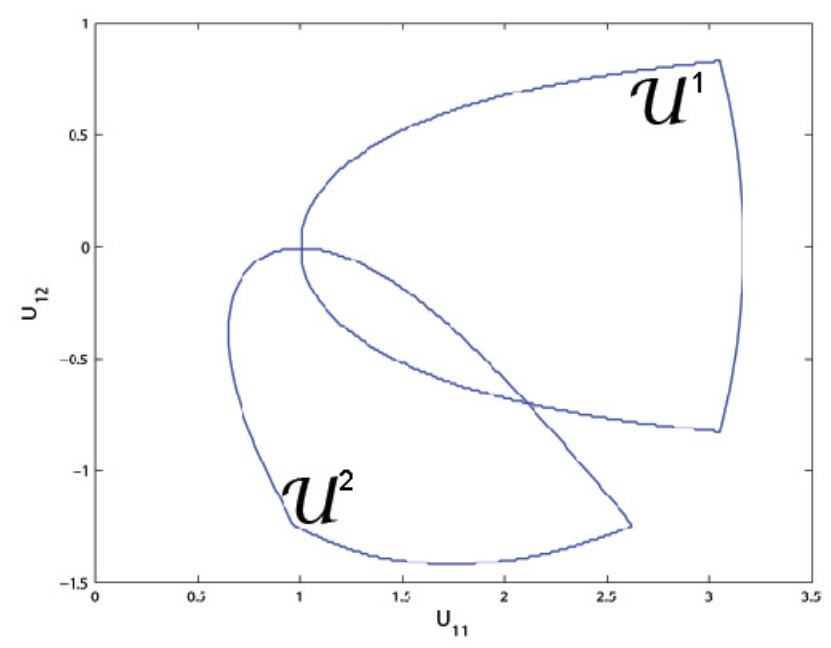

Fig. 1. Representation of the sets $\mathcal{U}_{1}$ and $\mathcal{U}_{2}$ for a two-orientation problem

As a consequence of the representation (4.14) and of the incompressibility condition $u_{11} u_{22}-u_{12}^{2}=1$, any tensor $\boldsymbol{U}$ in $\mathcal{U}_{1} \cup \mathcal{U}_{2}$ is entirely determined by its two components $u_{11}, u_{12}$. This allows one to give two-dimensional representations of the sets $\mathcal{U}_{1}$ and $\mathcal{U}_{2}$, as illustrated on Figure 1. On that Figure are represented the projections of the sets $\mathcal{U}_{1}$ and $\mathcal{U}_{2}$ in the plane $\left(u_{11}, u_{12}\right)$. The parameter $\delta$ is chosen equal to 3 .

On Figure 2, the solids lines show the boundary of the domain $\mathcal{U}^{+}(\boldsymbol{c})$, for several values of $c_{1}$. In particular, we can notice how the domain $\mathcal{U}^{+}(\boldsymbol{c})$ morphes from $\mathcal{U}_{2}$ to $\mathcal{U}_{1}$ as $c_{1}$ increases from 0 to 1 .

\section{Optimality of the bound $\mathcal{S}^{+}(c)$}

The bound $\mathcal{S}^{+}(\boldsymbol{c})$ is optimal if for any $\overline{\boldsymbol{F}} \in \mathcal{S}^{+}(\boldsymbol{c})$, there exists a sequence of microstructures - defined by characteristic functions $\chi_{1}^{i}, \cdots, \chi_{r}^{i}$ - and a bounded sequence of deformation gradient fields $\boldsymbol{F}^{i} \in \mathcal{K}(\overline{\boldsymbol{F}})$ such that

$$
\begin{gathered}
\int_{\Omega} \chi_{r}^{i}(\boldsymbol{x}) d \boldsymbol{x}=c_{r}(r=1, \cdots, n) \\
\operatorname{mes}\left\{\boldsymbol{x} \in \Omega \mid \boldsymbol{F}^{i}(\boldsymbol{x}) \notin \mathcal{S}(\boldsymbol{x})\right\} \longrightarrow 0 \text { as } i \longrightarrow+\infty
\end{gathered}
$$

Because of the decomposition (3.12), the optimality of $\mathcal{S}^{+}(\boldsymbol{c})$ is equivalent to the optimality of $\mathcal{U}^{+}(\boldsymbol{c})$, i.e. it is sufficient to verify the condition (5.17) for deformation gradients $\overline{\boldsymbol{F}}$ which are symmetric positive definite.

For the two-orientation problem considered in Section 4, we check the optimality of $\mathcal{S}^{+}(\boldsymbol{c})$ (or $\left.\mathcal{U}^{+}(\boldsymbol{c})\right)$ by considering simply laminated microstructures. 

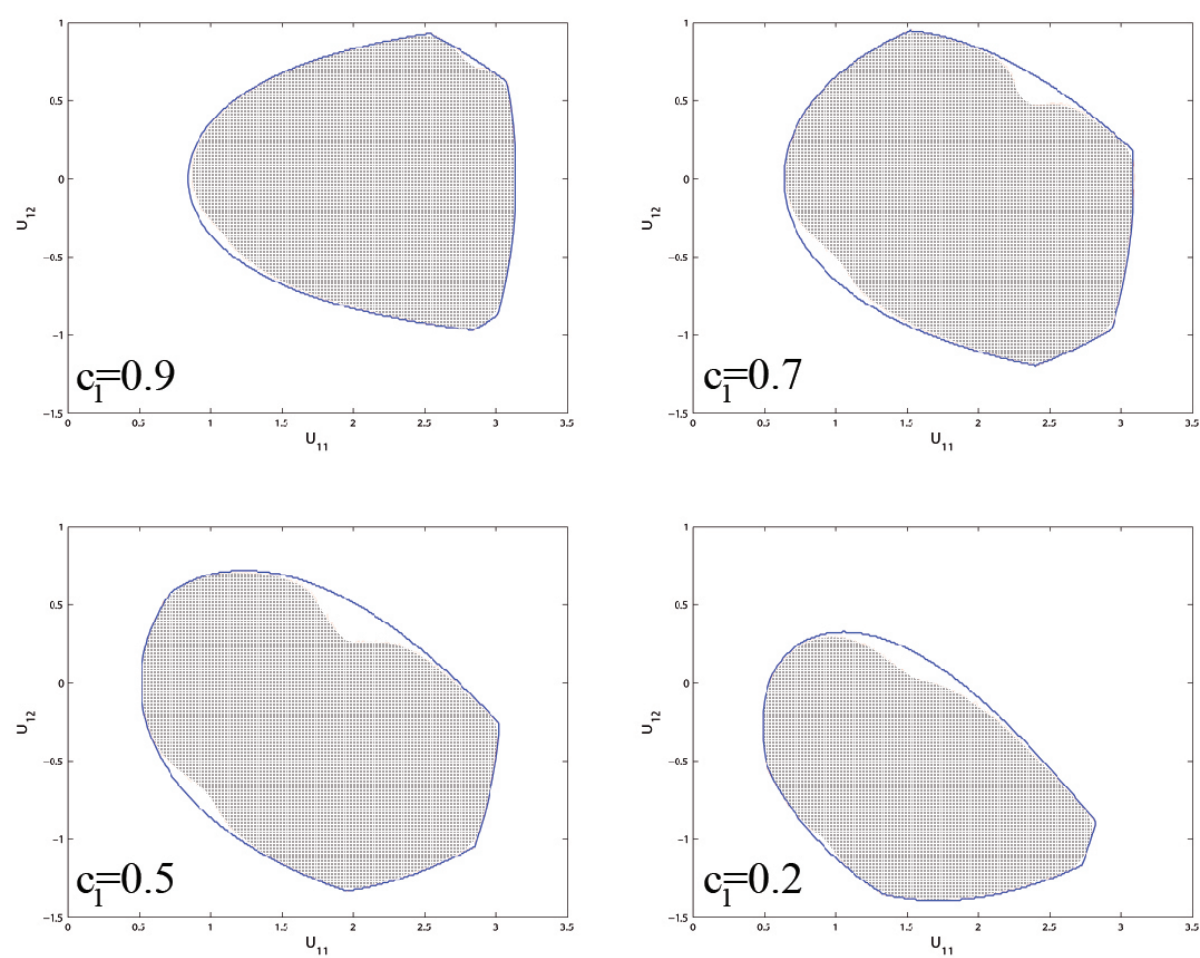

Fig. 2. The upper bound $\mathcal{U}^{+}(\boldsymbol{c})$ (delimited by solid lines) and the lower bound $\mathcal{U}^{-}(\boldsymbol{c})$ (dotted area) for several value of the volume fraction $c_{1}$.

In such microstructures, the characteristic functions $\chi_{r}(\boldsymbol{x})$ are of the form $\chi_{r}(\boldsymbol{x})=g_{r}(\boldsymbol{x} . \boldsymbol{N})$ where $\boldsymbol{N}$ is a vector (independent on $r$ ), corresponding to the normal to the interfaces in the laminate. The vector $\boldsymbol{N}$ is called the direction of lamination.

Let us recall some results on the theory of laminates in finite strains. Two given deformation gradients $\boldsymbol{F}_{1}$ and $\boldsymbol{F}_{2}$ are said to compatible (or rank-1 connected) if they verify

$$
\boldsymbol{F}_{1}-\boldsymbol{R} \boldsymbol{F}_{2}=\boldsymbol{b} \otimes \boldsymbol{n}
$$

for some rotation $\boldsymbol{R}$ and vectors $(\boldsymbol{b}, \boldsymbol{n})$. Setting $\boldsymbol{C}\left(\boldsymbol{F}_{1}, \boldsymbol{F}_{2}\right)=\boldsymbol{F}_{1}^{-T} \boldsymbol{F}_{2}^{T} \boldsymbol{F}_{2} \boldsymbol{F}_{1}^{-1}$ and denoting by $\lambda_{1} \leq \lambda_{2} \leq \lambda_{3}$ the eigenvalues of $\boldsymbol{C}\left(\boldsymbol{F}_{1}, \boldsymbol{F}_{2}\right)$, the following property can be proved (Ball and James, 1987):

$$
\boldsymbol{F}_{1} \text { and } \boldsymbol{F}_{2} \text { are compatible } \Longleftrightarrow \lambda_{2}=1 \text {. }
$$

Moreover, if $\boldsymbol{F}_{1}$ and $\boldsymbol{F}_{2}$ are compatible, the vectors $\boldsymbol{b}$ and $\boldsymbol{n}$ solutions of (5.18) are given by the following expressions:

$$
\begin{aligned}
& \boldsymbol{b}=\quad \sqrt{\frac{\lambda_{3}\left(1-\lambda_{1}\right)}{\lambda_{3}-\lambda_{1}}} \boldsymbol{u}_{1}+\kappa \sqrt{\frac{\lambda_{1}\left(\lambda_{3}-1\right)}{\lambda_{3}-\lambda_{1}}} \boldsymbol{u}_{3} \\
& \boldsymbol{n}=\frac{\sqrt{\lambda_{3}}-\sqrt{\lambda_{1}}}{\sqrt{\lambda_{3}-\lambda_{1}}}\left(-\sqrt{1-\lambda_{1}} \boldsymbol{F}_{2}^{T} \boldsymbol{u}_{1}+\kappa \sqrt{\lambda_{3}-1} \boldsymbol{F}_{2}^{T} \boldsymbol{u}_{3}\right)
\end{aligned}
$$


where $\kappa= \pm 1$, and $\boldsymbol{u}_{1}$ (resp. $\left.\boldsymbol{u}_{3}\right)$ is the eigenvector of $\boldsymbol{C}\left(\boldsymbol{F}_{1}, \boldsymbol{F}_{2}\right)$ associated to the eigenvalue $\lambda_{1}$ (resp. $\lambda_{3}$ ). Consider two compatible deformation gradients $\boldsymbol{F}_{1}$ and $\boldsymbol{F}_{2}$, and let $\left(\chi_{1}, \chi_{2}\right)$ be a laminate microstructure with a direction of lamination equal to the vector $\boldsymbol{n}$ in (5.18). It is well known that the deformation gradient field $\boldsymbol{F}(\boldsymbol{x})=\chi_{1}(\boldsymbol{x}) \boldsymbol{F}_{1}+\chi_{2}(\boldsymbol{x}) \boldsymbol{R} \boldsymbol{F}_{2}$ is admissible, i.e. there exists a transformation $\boldsymbol{u}: \Omega \mapsto \mathbb{R}^{3}$ such that $\nabla \boldsymbol{u}=\boldsymbol{F}$.

Those results can be used to study the optimality of the bound $\mathcal{U}^{+}(\boldsymbol{c})$. Take $\boldsymbol{U}_{1} \in \mathcal{U}_{1}$ and $\boldsymbol{U}_{2}$ in $\mathcal{U}_{2}$. The form (4.14) of $\boldsymbol{U}_{1}$ and $\boldsymbol{U}_{2}$ implies that 1 is eigenvalue of $\boldsymbol{C}\left(\boldsymbol{U}_{1}, \boldsymbol{U}_{2}\right)$, and that the two other eigenvalues $\lambda, \lambda^{\prime}$ verify $\lambda \lambda^{\prime}=$ 1. By (5.19), the deformation gradients $\boldsymbol{U}_{1}$ and $\boldsymbol{U}_{2}$ are compatible: there exists a rotation $\boldsymbol{R}^{\prime}$ and two vectors $\left(\boldsymbol{b}^{\prime}, \boldsymbol{n}^{\prime}\right)$ such that $\boldsymbol{U}_{1}-\boldsymbol{R}^{\prime} \boldsymbol{U}_{2}=\boldsymbol{b}^{\prime} \otimes \boldsymbol{n}^{\prime}$. Note by (5.20) that $\boldsymbol{n}^{\prime}$ is in the plane $\left(\boldsymbol{v}_{1}, \boldsymbol{v}_{2}\right)$. For an arbitrary value of $\boldsymbol{c}=\left(c_{1}, c_{2}\right)$, consider a simple laminate $\left(\chi_{1}, \chi_{2}\right)$ having a direction of lamination $\boldsymbol{n}^{\prime}$ and mixing orientations 1 and 2 in proportion $c_{1}$ and $c_{2}$ (i.e. $\int_{\Omega} \chi_{r}(\boldsymbol{x}) d \boldsymbol{x}=c_{r}$ for $r=1,2)$. The deformation gradient field $\boldsymbol{F}_{\chi}(\boldsymbol{x})=\chi_{1}(\boldsymbol{x}) \boldsymbol{U}_{1}+\chi_{2}(\boldsymbol{x}) \boldsymbol{R}^{\prime} \boldsymbol{U}_{2}$ is admissible and verifies the condition $\boldsymbol{F}_{\chi}(\boldsymbol{x}) \in \mathcal{S}(\boldsymbol{x})$ for all $\boldsymbol{x}$ in $\Omega$. Setting $\overline{\boldsymbol{F}}^{*}=$ $\boldsymbol{U}_{1}+c_{2} \boldsymbol{b}^{\prime} \otimes \boldsymbol{n}^{\prime}$, the deformation gradient field $\boldsymbol{F}_{\chi}$ satisfies the equality $\overline{\boldsymbol{F}}^{*}=$ $\int_{\Omega} \boldsymbol{F}_{\chi}(\boldsymbol{x}) d \boldsymbol{x}$, which by (3.9) is a necessary condition for $\boldsymbol{F}_{\chi}$ to be in $\mathcal{K}\left(\overline{\boldsymbol{F}}^{*}\right)$. However, $\boldsymbol{F}_{\chi}(\boldsymbol{x})$ is not necessarily equal to $\overline{\boldsymbol{F}}^{*} \boldsymbol{x}$ for $\boldsymbol{x} \in \partial \Omega$, and therefore it cannot be concluded directly that $\boldsymbol{F}_{\chi} \in \mathcal{K}\left(\overline{\boldsymbol{F}}^{*}\right)$ and that $\overline{\boldsymbol{F}}^{*}$ is recoverable. We actually need to consider a sequence of increasingly fine laminates $\left(\chi_{1}^{i}, \chi_{2}^{i}\right)$ - with a direction of lamination $\boldsymbol{n}^{\prime}$ and such that $\int_{\Omega} \chi_{r}^{i}(\boldsymbol{x}) d \boldsymbol{x}=c_{r}$ - and take the limit as $i \rightarrow \infty$. Reproducing arguments introduced by Ball and James (1992), it is possible to construct a bounded sequence of admissible deformation gradients $\boldsymbol{F}^{i}$ such that $\boldsymbol{F}^{i}(\boldsymbol{x})=\chi_{1}^{i}(\boldsymbol{x}) \boldsymbol{U}_{1}+\chi_{2}^{i}(\boldsymbol{x}) \boldsymbol{R}^{\prime} \boldsymbol{U}_{2}$ for all $\boldsymbol{x}$ in $\Omega$ except in a region $\Gamma^{i}$ surrounding the boundary $\partial \Omega$, the measure or $\Gamma^{i}$ tending to 0 as $i \longrightarrow \infty$. Therefore, by (5.17), it can be concluded that $\overline{\boldsymbol{F}}^{*}=\boldsymbol{U}_{1}+c_{2} \boldsymbol{b}^{\prime} \otimes \boldsymbol{n}^{\prime}$ is a recoverable strain. Since $\tilde{\mathcal{S}}$ respect the principle of frame indifference, the deformation gradient $\boldsymbol{R}^{*} \boldsymbol{F}^{*}$ is also recoverable, for any rotation $\boldsymbol{R}^{*}$. Consequently, the right-stretch tensor of $\boldsymbol{F}^{*}$ is recoverable.

Building on those considerations, we now describe a procedure for estimating the optimality of the upper bound $\mathcal{U}^{+}(\boldsymbol{c})$. Let $\boldsymbol{U}_{1}$ and $\boldsymbol{U}_{2}$ vary in $\mathcal{U}_{1} \times \mathcal{U}_{2}$. In practice, the parametrization of $\mathcal{U}_{1}$ and $\mathcal{U}_{2}$ is easily achieved using the expressions (4.15)-(4.16). For any $\left(\boldsymbol{U}_{1}, \boldsymbol{U}_{2}\right)$, calculate the vectors $\boldsymbol{b}$ and $\boldsymbol{n}$ given by the expressions (5.20). The arguments developed above show that the right stretch tensor of $\boldsymbol{U}_{1}+c_{2} \boldsymbol{b} \otimes \boldsymbol{n}$ is recoverable, for any volume fractions $\left(c_{1}, c_{2}\right)$.

That algorithm allows one to construct a domain $\mathcal{U}^{-}(\boldsymbol{c})$ of recoverable strains that can be realized by simply laminated microstructures. That domain $\mathcal{U}^{-}(\boldsymbol{c})$ is represented as a dotted area on Figure 2, for several values of the volume fraction $c_{1}$. As can be observed on Figure 2, the gap between $\mathcal{U}^{-}(\boldsymbol{c})$ and $\mathcal{U}^{+}(\boldsymbol{c})$ is relatively small. This means that the bound $\mathcal{U}^{+}(\boldsymbol{c})$ is close to being optimal, 
at least for the two-orientation problem considered. In that regard, it should be mentioned that the construction of laminate microstructures can be developed further, by considering second- or higher-rank laminates (Milton, 2002). Such a construction would improve the conclusions regarding the optimality of the bound $\mathcal{U}^{+}(\boldsymbol{c})$ : the set $\mathcal{U}^{*}(\boldsymbol{c})$ of recoverable strains that can be realized by second- or higher-rank laminates is larger that $\mathcal{U}^{-}(\boldsymbol{c})$ and therefore closer to $\mathcal{U}^{+}(\boldsymbol{c})$.

\section{Concluding remarks}

New upper bounds on the set of recoverable strains have been presented, in the framework of finite deformations. At present stage, those upper bounds only involve first-order statistics of the polycrystalline texture, i.e. the volume fractions of the different orientations. The optimality of such bounds has been estimated on a reference two-orientation problem: the consideration of simply laminated textures has allowed us to verify that the upper bound presented is tight. This is an encouraging sign, and it would now be interesting to carry out a similar investigation for more complex textures and materials. An expected difficulty lies in the fact that, in finite strains, the set $\mathcal{S}^{0}$ is not known explicitly for single crystals with more than two variants of martensite. Some investigation is under way to solve that problem. An other line of investigation consists in studying bounds that take two- or higher-order statistical information into account. In infinitesimal strains, this has been proved to be possible for the related problem of bounding the effective free energy of martensitic polycrystals (Peigney, 2009). The extension of such results to the finite strains setting is not straightforward and remains to explore.

\section{References}

Ball J.M., James R.D., Fine phase mixtures as minimizers of energy, Arch.Rat.Mech.Analysis, 100, p.13-52, 1987.

Ball J.M., James R.D., Proposed experimental tests of a theory of fine microstructure and the two-well problem, Phil.Trans.Roy.Soc.London A., 338, p.338-450, 1992.

Bhattacharya K., Kohn R., Energy minimization recoverable strains polycrystalline shape memory alloys, Arch.Rat.Mech.Analysis, 139, 2, p.99-180, 1997.

Bhattacharya K., Microstructure of martensite, Oxford University Press, 2003. Dacorogna B., Direct methods in the calculus of variations, Springer, 1989.

Milton G., Serkov S., Bounding the current in nonlinear conducting composites, J.Mech.Phys.Solids, 48, p.1295-1324, 2000. 
Milton G., The theory of composites, Cambridge University Press, 2002.

Peigney M., A pattern-based method for bounding the effective response of a nonlinear composite, J.Mech.Phys.Solids, 53, p.923-948, 2005.

Peigney M., Recoverable strains in composite shape-memory alloys, J.Mech.Phys.Solids, 56, p.360-375, 2008.

Peigney M., A non-convex lower bound on the effective energy of polycrystalline shape-memory alloys, J.Mech.Phys.Solids, 57, p.970-986, 2009.

Shu Y., Bhattacharya K., The influence of texture on the shape memory effect in polycrystals, Acta Mater., 46, p.5457-5473, 1998.

Talbot D.R.S., Willis J.R., Bounds for the effective constitutive relation of a nonlinear composite, Proceedings of the Royal Society, 460, p.2705-2723, 2004 . 